\title{
Fuzzification functions for temporal intuitionistic fuzzy sets
}

\author{
R. Parvathi ${ }^{1}$ and C. Radhamani ${ }^{2}$ \\ ${ }^{1}$ Department of Mathematics, Vellalar College for Women (Autonomous) \\ Erode - 638 107, Tamil Nadu, India \\ e-mail: paarvathiserediffmail.com \\ ${ }^{2}$ Department of Mathematics, Kongu Arts and Science College (Autonomous) \\ Erode - 638 012, Tamil Nadu, India \\ e-mail: palaniradhu@gmail.com
}

Received: 24 May 2019 Revised: 9 September $2019 \quad$ Accepted: 17 September 2019

\begin{abstract}
Fuzzification functions play a vital role in the development of fuzzy sets which handle uncertainty and vagueness more efficiently than Cantorian sets. In this paper, some standard fuzzification functions for temporal intuitionistic fuzzy sets are defined which are useful to deal with dynamical systems with uncertainty. The paper describes some standard models to explicitly incorporate time as a variable through which temporal reference of the object is included. Further, the so defined functions are illustrated with suitable examples.
\end{abstract}

Keywords: Intuitionistic fuzzy sets, Temporal intuitionistic fuzzy sets, Temporal intuitionistic fuzzification functions.

2010 Mathematics Subject Classification: $03 E 72$.

\section{Introduction}

Fuzzy mathematics, introduced by L. A. Zadeh in 1965, [14], has its own importance due to the inherent fuzziness occurring in nature. Uncertainty is most commonly prevalent in real life because bulk of information available every day is fuzzy. Uncertainty could be addressed with fuzzy sets (FSs) where the exact crisp inputs are transformed into fuzzy inputs and fuzzy control rules are applied to get the solution which is defuzzified to obtain a crisp output. FSs allow partial 
membership values ranging from 0 to 1 , unlike crisp sets where the membership of the element considered can be either 0 or 1 . Since it is not always certain that non-membership gives the exact complement of the membership values to $1, \mathrm{~K}$. T. Atanassov defined intuitionistic fuzzy sets (IFSs) which include non-membership and the hesitation degree as second and third factors [1-3]. He also defined temporal intuitionistic fuzzy sets (TIFSs) with time factor as one of the domains.

In [4-8], the authors introduced intuitionistic fuzzy multidimensional sets (IFMDSs), a new extension of IFSs, as well as of TIFSs, by changing the temporal component with an $n$-dimensional vector. In [11], again TIFSs are extended to multi-dimensional temporal intuitionistic fuzzy sets (MTIFSs). In order to apply the different fuzzy concepts, defining fuzzification and defuzzification methods play a vital role. The first and foremost step in fuzzy mathematics is defining suitable fuzzification functions (membership functions). The information in real life is fuzzy and the decisions to be implemented are either crisp or binary. Fuzzification is capable of supplying the inputs in terms of fuzzy sets where defuzzification provides the output in crisp terminology. A membership function is attached to each element of the crisp set to be fuzzified. The process of changing a real scalar value into a fuzzy value is fuzzification using fuzzification functions (fuzzifiers or membership functions) which can not be common as they vary from problem to problem. The fuzzification functions gave rise to fuzzy sets represented by fuzzy linguistic variables and deal with fuzzy information having its crisp counterpart in hand. Although an exact system may achieve a better performance, it takes much time for execution. Hence, there are some standard fuzzification functions like triangular, trapezoidal, Gaussian, bell-shaped, sigmoidal, S-shaped and Z-shaped functions available in literature which may obtain a good result with minimum effort and error. Being built from basic functions, they possess easily adjustable characteristics and have the advantage of simplicity and better accuracy. Some popular intuitionistic fuzzification functions with hesitancy are discussed in [12]. In [9] and [10], new formulae for de-i-fuzzification are developed. This paper provides various types of temporal intuitionistic fuzzification functions (membership and non-membership functions) of TIFSs which are effective in constructing temporal intuitionistic fuzzy logic systems/controllers.

The remaining part of the paper is organized as follows. Some basic concepts and definitions of IFSs, TIFSs and fuzzification functions of IFSs are overviewed in Section 2. In Section 3, some standard fuzzification functions for TIFS are presented and Section 4 describes suitable illustrations to identify the need for fuzzifiction functions in TIFSs. Finally, Section 5 concludes the paper.

\section{Preliminaries}

In this section, some prerequisites which are needed for this paper are overviewed.

Definition 2.1 ([1]). Let $X$ be a non-empty set. An intuitionistic fuzzy set (IFS) $A$ in $X$ is an object having the form $A=\left\{\left\langle x, \mu_{A}(X), \nu_{A}(X)\right\rangle / x \in X\right\}$ where $\mu_{A}: X \rightarrow[0,1]$ and $\nu_{A}: X \rightarrow[0,1]$ with $0 \leq \mu_{A}(x)+\nu_{A}(x) \leq 1$ for all $x \in X$ represent, respectively, the degrees of membership and non-membership of the element $x$ to the IFS A. For each IFS, the intuitionistic index or hesitancy degree of $x$ in $X$ to the IFS $A$ is $\pi_{A}(x)=1-\mu_{A}(x)-\nu_{A}(x)$. 
Definition 2.2 ([3]). Let $E$ be the universal set and $T$ be a non-empty set of time moments. Then, a temporal intuitionistic fuzzy set (TIFS) is an object having the form

$$
A(T)=\left\{\left\langle x, \mu_{A}(x, t), \nu_{A}(x, t)\right\rangle /(x, t) \in E \times T\right\}
$$

where

1. $A \subset E$ is a fixed set.

2. $\mu_{A}(x, t)$ and $\nu_{A}(x, t)$ denote the degrees of membership and non-membership, respectively, of the element $(x, t)$ such that $0 \leq \mu_{A}(x, t)+\nu_{A}(x, t) \leq 1$ for all $(x, t) \in E \times T$.

Definition 2.3 ([4]). Let the sets $Z_{1}, Z_{2}, \ldots, Z_{n}$ be fixed and let for each $i(1 \leq i \leq n)$ : $z_{i} \in Z_{i}$. Let the set $E$ be fixed. Then, an intuitionistic fuzzy multidimensional set IFMDS $A$ in $E \times Z_{1} \times Z_{2} \times \cdots \times Z_{n}$ is an object of the form

$$
\begin{aligned}
A\left(Z_{1}, Z_{2}, \ldots, Z_{n}\right)= & \left\{\left\langle x, \mu_{A}\left(x, z_{1}, z_{2}, \ldots, z_{n}\right), \nu_{A}\left(x, z_{1}, z_{2}, \ldots, z_{n}\right)\right\rangle \mid\left(x, z_{1}, z_{2}, \ldots, z_{n}\right) \in E\right. \\
& \left.\times Z_{1} \times Z_{2} \times \cdots \times Z_{n}\right\}
\end{aligned}
$$

where

(i) $\mu_{A}\left(x, z_{1}, z_{2}, \ldots, z_{n}\right)+\nu_{A}\left(x, z_{1}, z_{2}, \ldots, z_{n}\right) \leq 1$ for every $\left(x, z_{1}, z_{2}, \ldots, z_{n}\right) \in E \times Z_{1} \times$ $Z_{2} \times \cdots \times Z_{n}$

(ii) $\mu_{A}\left(x, z_{1}, z_{2}, \ldots, z_{n}\right)$ and $\nu_{A}\left(x, z_{1}, z_{2}, \ldots, z_{n}\right)$ are the degrees of membership and nonmembership respectively, of the element $\left(x, z_{1}, z_{2}, \ldots, z_{n}\right) \in E \times Z_{1} \times Z_{2} \times \cdots \times Z_{n}$.

Definition 2.4 ([11]). Let $E$ be the universe, $T$ be a non-empty set of time moments and $P=\left(P_{1}, P_{2}, \cdots P_{n}\right), P_{i}, i=1,2, \ldots, n$ be distinct sets of parameters which $E$ depends on. Let $p$ be an $n$-tuple $\left(p_{1}, p_{2}, \cdots p_{n}\right)$, where $p_{i} \in P_{i}, i=1,2, \ldots, n$. Then a multi-parameter temporal intuitionistic fuzzy set (MTIFS) defined on $E$ is an object of the form

$$
A(T, P)=\left\{\left\langle x, \mu_{A}(x, t, p), \nu_{A}(x, t, p)\right\rangle \mid(x, t, p) \in E \times T \times \prod_{i=1}^{n} P_{i}\right\}
$$

where

(i) $A \subset E$ is a fixed set.

(ii) $\mu_{A}(x, t, p)$ and $\nu_{A}(x, t, p)$ denote the degrees of membership and non-membership, respectively, of the element $(x, t, p) \in E \times T \times \prod_{i=1}^{n} P_{i}$ such that $0 \leq \mu_{A}(x, t, p)+\nu_{A}(x, t, p) \leq 1$ for all $(x, t, p) \in E \times T \times \prod_{i=1}^{n} P_{i}$.

Definition 2.5 ([13]). Membership function for an intuitionistic fuzzy set $A$ on the universe of discourse $X$ is defined as $\mu_{A}: X \rightarrow[0,1]$, where each element $x$ is mapped to a value between 0 and 1 . The value $\mu_{A}(x), x \in X$ is called the membership value or degree of membership of the element $x$ to the IFS A.

Definition 2.6 ([13]). Non-membership function for an intuitionistic fuzzy set $A$ on the universe of discourse $X$ is defined as $\nu_{A}: X \rightarrow[0,1]$, where each element $x$ is mapped to a value between 0 and 1 . The value $\nu_{A}(x), x \in X$ is called the non-membership value or degree of non-membership of the element $x$ to the IFS A. 


\section{Some standard fuzzification functions of TIFSs}

Fuzzification is the process of characterizing the fuzziness incorporated within the problem in order to convert the existing crisp quantity into a fuzzy value or intuitionistic fuzzy value. Fuzzification is done by defining membership functions which may vary depending on the nature of the problem. Likewise, intuitionistic fuzzification functions can be defined which include the membership and non-membership functions along with a hesitancy margin. Many theoretical and practical problems need different fuzzification functions in order to handle them in fuzzy for achieving maximum level of accuracy. Though it is not possible to find a unique representation of fuzzification functions, some standard popular functions for specifying FSs and IFSs which nearly satisfy the needs of the problem are available. The similar case for TIFS without hesitancy entities is dealt in [11]. This section discusses the formulation of some of the generalized fuzzification functions like triangular, trapezoidal, Gaussian, bell-shaped, sigmoidal, $S$-shaped and $Z$-shaped for TIFSs with hesitation margin.

Note: Through out this section, Let $X$ be the universe, $T$ be a non-empty set of time moments and $A(T)$ be the corresponding temporal intuitionistic fuzzy set defined on $E$ and $T$. The hesitancy degree denoted as $\epsilon$ can either be a suitably chosen fixed parameter or it can be a least fraction of the membership and non-membership values depending on the choice of the problem.

\subsection{Temporal triangular intuitionistic fuzzification functions}

The temporal triangular intuitionistic fuzzification functions for the TIFS $A(T)$ with a degree of hesitancy $\epsilon$ are defined as follows:

$$
\mu_{A}(x, t)= \begin{cases}\frac{x+t-(a+c)}{m+m^{\prime}-(a+c)}-\frac{\epsilon}{2}, & a<x \leq m, c<t<m^{\prime} \\ \frac{x+t-\left(a+m^{\prime}\right)}{m+d-\left(a+m^{\prime}\right)}-\frac{\epsilon}{2}, & a<x \leq m, m^{\prime} \leq t<d \\ \frac{b+m^{\prime}-(x+t)}{b+m^{\prime}-(m+c)}-\frac{\epsilon}{2}, & m<x<b, c<t<m^{\prime} \\ \frac{b+d-(x+t)}{b+d-\left(m+m^{\prime}\right)}-\frac{\epsilon}{2}, & m<x<b, m^{\prime} \leq t<d \\ 0, & \text { otherwise }\end{cases}
$$

and

$$
\nu_{A}(x, t)= \begin{cases}\frac{m+m^{\prime}-(x+t)}{m+m^{\prime}-(a+c)}-\frac{\epsilon}{2}, & a<x \leq m, c<t<m^{\prime} \\ \frac{m+d-(x+t)}{m+d-\left(a+m^{\prime}\right)}-\frac{\epsilon}{2}, & a<x \leq m, m^{\prime} \leq t<d \\ \frac{x+t-(m+c)}{b+m^{\prime}-(m+c)}-\frac{\epsilon}{2}, & m<x<b, c<t<m^{\prime} \\ \frac{x+t-\left(m+m^{\prime}\right)}{b+d-\left(m+m^{\prime}\right)}-\frac{\epsilon}{2}, & m<x<b, m^{\prime} \leq t<d \\ 1-\epsilon, & \text { otherwise }\end{cases}
$$

where $a \leq x \leq b, a<m<b, c \leq t \leq d$ and $c<m^{\prime}<d$.

Remark 1. When $T$ is a singleton set, the triangular intuitionistic fuzzification functions for TIFSs is equal to triangular intuitionistic fuzzification functions for IFSs and when $\epsilon=0$ and $T$ is a singleton set, it becomes a triangular fuzzification function. This fact holds true for the all the fuzzification functions to be defined in the rest of the section. 


\subsection{Temporal trapezoidal intuitionistic fuzzification functions}

The temporal trapezoidal intuitionistic fuzzification functions for the TIFS $A(T)$ are defined as

$$
\mu_{A}(x, t)= \begin{cases}\frac{x+t-(a+c)}{m_{1}+m_{1}^{\prime}-(a+c)}-\frac{\epsilon}{2}, & a<x<m_{1}, c<t<m_{1}^{\prime} \\ \frac{x+t-\left(a+m_{1}^{\prime}\right)}{m_{1}+m_{2}^{\prime}-\left(a+m_{1}^{\prime}\right)}-\frac{\epsilon}{2}, & a<x<m_{1}, m_{1}^{\prime}<t<m_{2}^{\prime} \\ \frac{x+t-\left(a+m_{2}^{\prime}\right)}{m_{1}+d-\left(a+m_{2}^{\prime}\right)}-\frac{\epsilon}{2}, & a<x<m_{1}, m_{2}^{\prime}<t<d \\ 1-\epsilon, & m_{1} \leq x \leq m_{2} / m_{1}^{\prime} \leq t \leq m_{2}^{\prime} \\ \frac{b+m_{1}^{\prime}-(x+t)}{b+m_{1}^{\prime}-\left(m_{2}+c\right)}-\frac{\epsilon}{2}, & m_{2}<x<b, c<t<m_{1}^{\prime} \\ \frac{b+m_{2}^{\prime}-(x+t)}{b+m_{2}^{\prime}-\left(m_{2}+m_{1}^{\prime}\right)}, & m_{2}<x<b, m_{1}^{\prime}<t<m_{2}^{\prime} \\ \frac{b+d-(x+t)}{b+d-\left(m_{2}+m_{2}^{\prime}\right)}-\frac{\epsilon}{2}, & m_{2}<x<b, m_{2}^{\prime}<t<d \\ 0, & \text { otherwise }\end{cases}
$$

and

$$
\nu_{A}(x, t)= \begin{cases}1-\frac{x+t-(a+c)}{m_{1}+m_{1}^{\prime}-(a+c)}-\frac{\epsilon}{2}, & a<x<m_{1}, c<t<m_{1}^{\prime} \\ 1-\frac{x+t-\left(a+m_{1}^{\prime}\right)}{m_{1}+m_{2}^{\prime}-\left(a+m_{1}^{\prime}\right)}-\frac{\epsilon}{2}, & a<x<m_{1}, m_{1}^{\prime}<t<m_{2}^{\prime} \\ 1-\frac{x+t-\left(a+m_{2}^{\prime}\right)}{m_{1}+d-\left(a+m_{2}^{\prime}\right)}-\frac{\epsilon}{2}, & a<x<m_{1}, m_{2}^{\prime}<t<d \\ 0, & m_{1} \leq x \leq m_{2} / m_{1}^{\prime} \leq t \leq m_{2}^{\prime} \\ 1-\frac{b+m_{1}^{\prime}-(x+t)}{b+m_{1}^{\prime}-\left(m_{2}+c\right)}-\frac{\epsilon}{2}, & m_{2}<x<b, c<t<m_{1}^{\prime} \\ 1-\frac{b+m_{2}^{\prime}-(x+t)}{b+m_{2}^{\prime}-\left(m_{2}+m_{1}^{\prime}\right)-\frac{\epsilon}{2}}, & m_{2}<x<b, m_{1}^{\prime}<t<m_{2}^{\prime} \\ 1-\frac{b+d-(x+t)}{b+d-\left(m_{2}+m_{2}^{\prime}\right)}-\frac{\epsilon}{2}, & m_{2}<x<b, m_{2}^{\prime}<t<d \\ 1-\epsilon, & \text { otherwise }\end{cases}
$$

where $a \leq x \leq b ; a<m_{1}, m_{2}<b ; c \leq t \leq d$ and $c<m_{1}^{\prime}, m_{2}^{\prime}<d$.

\subsection{Temporal R-trapezoidal intuitionistic fuzzification functions}

The temporal R-trapezoidal intuitionistic fuzzification functions for the TIFS $A(T)(b, d \rightarrow \infty)$ are defined as

$$
\mu_{A}(x, t)= \begin{cases}1-\epsilon, & x \leq a, t \leq c \\ \frac{(a+d)-(x+t)}{(a+d)-(x+c)}-\frac{\epsilon}{2}, & x \leq a, c<t<d \\ \frac{(b+c)-(x+t)}{(b+d)-(a+c)}-\frac{\epsilon}{2}, & a<x<b, c<t<d \\ \frac{(b+c)-(x+t)}{(b+d)-(a+t)}-\frac{\epsilon}{2}, & a<x<b, t \leq c \\ 0, & \text { otherwise }\end{cases}
$$


and

$$
\nu_{A}(x, t)= \begin{cases}0, & x \leq a, t \leq c \\ \frac{(x+t)-(x+c)}{(a+d)-(x+c)}-\frac{\epsilon}{2}, & x \leq a, c<t<d \\ \frac{(x+t)-(a+c)}{(b+d)-(a+c)}-\frac{\epsilon}{2}, & a<x<b, c<t<d \\ \frac{(x+t)-(a+t)}{(b+c)-(a+t)}-\frac{\epsilon}{2}, & a<x<b, t \leq c \\ 1-\epsilon, & \text { otherwise }\end{cases}
$$

where $a \leq b$ and $c \leq d$.

\subsection{Temporal L-trapezoidal intuitionistic fuzzification functions}

The temporal L-trapezoidal intuitionistic fuzzification functions for the TIFS $A(T)(a, c \rightarrow-\infty)$ are defined as

$$
\begin{gathered}
\mu_{A}(x, t)= \begin{cases}1-\epsilon, & x \geq b, t \geq d \\
\frac{x+t-(b+c)}{x+d-(b+c)}-\frac{\epsilon}{2}, & x \geq b, c<t<d \\
\frac{b+d-(x+t)}{b+d-(a+c)}-\frac{\epsilon}{2}, & a<x<b, c<t<d \\
\frac{b+t-(x+d)}{b+t-(a+d)}-\frac{\epsilon}{2}, & a<x<b, t \geq d \\
0, & \text { otherwise }\end{cases} \\
\nu_{A}(x, t)= \begin{cases}0, & x \geq b, t \geq d / c<t<d \\
\frac{(x+d)-(x+t)}{(x+d)-(b+c)}-\frac{\epsilon}{2}, & x \geq b, c<t<d \\
\frac{(x+t)-(a+c)}{(b+d)-(a+c)}-\frac{\epsilon}{2}, & a<x<b, c<t<d \\
\frac{(x+d)-(a+d)}{(b+t)-(a+d)}-\frac{\epsilon}{2}, & a<x<b, t \geq d \\
1-\epsilon, & \text { otherwise }\end{cases}
\end{gathered}
$$

where $a \leq x \leq b, a<b, c \leq t \leq d$ and $c<d$.

\subsection{Temporal Gaussian intuitionistic fuzzification functions}

The temporal Gaussian intuitionistic fuzzification functions for the TIFS $A(T)(a, c \rightarrow-\infty)$ are defined by the mean value $c$ (centre) of $X$, the standard deviation $k$ (width) of $X$ and the fuzzification factor $m$. Let the corresponding values of $T$ be $c^{\prime}, k^{\prime}$. The standard deviation values $k, k^{\prime}$ determine the narrowness. Gaussian membership and non-membership functions are defined as follows:

$$
\mu_{A}(x, t)=\exp \left(-\frac{\left((x+t)-\left(c+c^{\prime}\right)\right)^{m}}{2\left(k+k^{\prime}\right)^{m}}\right)-\frac{\epsilon}{2}
$$

and

$$
\nu_{A}(x, t)=1-\exp \left(-\frac{\left((x+t)-\left(c+c^{\prime}\right)\right)^{m}}{2\left(k+k^{\prime}\right)^{m}}\right)-\frac{\epsilon}{2}
$$




\subsection{Temporal bell-shaped intuitionistic fuzzification functions}

The temporal bell-shaped intuitionistic fuzzification functions for the TIFS $A(T)$ are specified by the six parameters $a, a^{\prime}$ (width of $X, T$ ), $c, c^{\prime}$ (centers of $X, T$ ) and $b, b^{\prime}$ (responsible for slopes). The temporal bell-shaped membership and non-membership functions are defined as follows:

$$
\mu_{A}(x, t)=\frac{1}{1+\left|\frac{(x+t)-\left(c+c^{\prime}\right)}{\left(a+a^{\prime}\right)}\right|^{2\left(b+b^{\prime}\right)}}-\frac{\epsilon}{2}
$$

and

$$
\nu_{A}(x, t)=1-\frac{1}{1+\left|\frac{(x+t)-\left(c+c^{\prime}\right)}{\left(a+a^{\prime}\right)}\right|^{2\left(b+b^{\prime}\right)}}-\frac{\epsilon}{2}
$$

\subsection{Temporal sigmoidal intuitionistic fuzzification functions}

The temporal sigmoidal intuitionistic fuzzification functions for the TIFS $A(T)$ are specified by the parameters $a, a^{\prime}$ which are responsible for slopes at the cross over points $\left(c, c^{\prime}\right)$. The positive and negative values of $a, a^{\prime}$ determines the position of the graphics. The temporal sigmoidal membership and non-membership functions for the TIFS $A(T)$ are defined as follows:

and

$$
\mu_{A}(x, t)=\left(\frac{1}{1+\exp \left(-\left(a+a^{\prime}\right)\left((x+t)-\left(c+c^{\prime}\right)\right)\right)}\right)-\frac{\epsilon}{2}
$$

$$
\nu_{A}(x, t)=1-\left(\frac{1}{1+\exp \left(-\left(a+a^{\prime}\right)\left((x+t)-\left(c+c^{\prime}\right)\right)\right)}\right)-\frac{\epsilon}{2}
$$

\subsection{Temporal S-shaped intuitionistic fuzzification functions}

The temporal S-shaped intuitionistic fuzzification functions for the TIFS $A(T)(a, c \rightarrow-\infty)$ are defined as

$$
\mu_{A}(x, t)= \begin{cases}1-\epsilon, & x \geq b, t \geq d \\ 2\left(\frac{x+t-(a+c)}{b+d-(a+c)}\right)^{2}-\frac{\epsilon}{2}, & a<x \leq \frac{a+b}{2}, c<t \leq \frac{c+d}{2} \\ 2\left(\frac{x+t-\left(a+\frac{c+d}{2}\right)}{b+d-(a+c)}\right)^{2}-\frac{\epsilon}{2}, & a<x \leq \frac{a+b}{2}, \frac{c+d}{2} \leq t<d \\ 1-2\left(\frac{x+t-\left(b+\frac{c+d}{2}\right.}{b+\frac{c+d}{2}-(a+c)}\right)^{2}-\frac{\epsilon}{2}, & \frac{a+b}{2} \leq x<b, c<t \leq \frac{c+d}{2} \\ 1-2\left(\frac{x+t-(b+d)}{b+d-(a+c)}\right)-\frac{\epsilon}{2}, & \frac{a+b}{2} \leq x<b, \frac{c+d}{2} \leq t<d \\ 0, & x \leq a, t \leq c\end{cases}
$$

and

$$
\nu_{A}(x, t)= \begin{cases}0, & x \geq b, t \geq d \\ 1-2\left(\frac{x+t-(a+c)}{b+d-(a+c)}\right)^{2}-\frac{\epsilon}{2}, & a<x \leq \frac{a+b}{2}, c<t \leq \frac{c+d}{2} \\ 1-2\left(\frac{x+t-\left(a+\frac{c+d}{2}\right)}{b+d-(a+c)}\right)^{2}-\frac{\epsilon}{2}, & a<x \leq \frac{a+b}{2}, \frac{c+d}{2} \leq t<d \\ 2\left(\frac{x+t-\left(b+\frac{c+d}{2}\right.}{b+\frac{c+d}{2}-(a+c)}\right)^{2}-\frac{\epsilon}{2}, & \frac{a+b}{2} \leq x<b, c<t \leq \frac{c+d}{2} \\ 2\left(\frac{x+t-(b+d)}{b+d-(a+c)}\right)-\frac{\epsilon}{2}, & \frac{a+b}{2} \leq x<b, \frac{c+d}{2} \leq t<d \\ 1-\epsilon, & x \leq a, t \leq c\end{cases}
$$

where $a \leq x \leq b, a<b, c \leq t \leq d$ and $c<d$. 


\subsection{Temporal Z-shaped intuitionistic fuzzification functions}

The temporal Z-shaped intuitionistic fuzzification functions for the TIFS $A(T)(a, c \rightarrow-\infty)$ are defined as

$$
\mu_{A}(x, t)= \begin{cases}1-\epsilon, & x \geq b, t \geq d \\ 1-2\left(\frac{(x+t)-(a+c)}{(b+d)-(a+c)}\right)^{2}-\frac{\epsilon}{2}, & a<x \leq \frac{a+b}{2}, c<t \leq \frac{c+d}{2} \\ 1-2\left(\frac{(x+t)-\left(a+\frac{c+d}{2}\right.}{(b+d)-\left(a+\frac{c+d}{2}\right.}\right)^{2}-\frac{\epsilon}{2}, & a<x \leq \frac{a+b}{2}, \frac{c+d}{2} \leq t<d \\ 2\left(\frac{(x+t)-(b+d)}{(b+d)-(a+c)}\right)^{2}-\frac{\epsilon}{2}, & \frac{a+b}{2} \leq x<b, c<t \leq \frac{c+d}{2} \\ 2\left(\frac{(x+t)-(b+d)}{(b+d)-(a+c)}\right)-\frac{\epsilon}{2}, & \frac{a+b}{2} \leq x<b, \frac{c+d}{2} \leq t<d \\ 0, & x \leq a, t \leq c\end{cases}
$$

and

$$
\nu_{A}(x, t)= \begin{cases}0, & x \geq b, t \geq d \\ 2\left(\frac{(x+t)-(a+c)}{(b+d)-(a+c)}\right)^{2}-\frac{\epsilon}{2}, & a<x \leq \frac{a+b}{2}, c<t \leq \frac{c+d}{2} \\ 2\left(\frac{(x+t)-\left(a+\frac{c+d}{2}\right.}{(b+d)-\left(a+\frac{c+d}{2}\right.}\right)^{2}-\frac{\epsilon}{2}, & a<x \leq \frac{a+b}{2}, \frac{c+d}{2} \leq t<d \\ 1-2\left(\frac{(x+t)-(b+d)}{(b+d)-(a+c)}\right)^{2}-\frac{\epsilon}{2}, & \frac{a+b}{2} \leq x<b, c<t \leq \frac{c+d}{2} \\ 1-2\left(\frac{(x+t)-(b+d)}{(b+d)-(a+c)}\right)-\frac{\epsilon}{2}, & \frac{a+b}{2} \leq x<b, \frac{c+d}{2} \leq t<d \\ 1-\epsilon, & x \leq a, t \leq c\end{cases}
$$

where $a \leq x \leq b, a<b, c \leq t \leq d$ and $c<d$.

\section{Illustration and discussion}

This section describes the representation of temporal intuitionistic fuzzification functions in discrete and continuous time domains. Suitable illustrations are considered and discussed to explain the necessity of having standard types of temporal intuitionistic fuzzification functions. Temporal intuitionistic fuzzy systems which are capable of providing a better performance in real environments are constructed.

Example 1. Consider a patient with symptoms related to diabetes consulting a physician for diagnosis. The doctor needs the past history of the patient including the diabetic data of the patient taken over some prior period along with a set of continuous observations in order to strongly conclude whether he is a diabetic or not. Since Blood Glucose Levels (BGLs) of the patient determine the diabetic level, BGLs are taken over some consecutive period. As per the diabetic medical knowledge is concerned, if the BGL lies between 80 to 140, 140 to 300 and above 300 , it is understood that the patient is a non-diabetic, Type I diabetic and Type II diabetic respectively. Let the universal set $X$ be the set of BGLs and a discrete time domain $T=\{1,2,3\}$ be considered in days. That is, the BGLs of the patient under investigation on three consecutive days. The three sets non-diabetic, Type I diabetic and Type II diabetic can be represented as TIFSs by defining the corresponding temporal intuitionistic fuzzification functions. 
From the fuzzification functions defined in Section 3.1, the temporal triangular fuzzification functions for the TIFS Type I diabetic are defined as follows:

$$
\mu(x, t)= \begin{cases}\frac{x+t-(121)}{101}-\frac{\epsilon}{2}, & 120<x \leq 220,1<t<2 \\ \frac{x+t-(122)}{101}-\frac{\epsilon}{2}, & 120<x \leq 220,2 \leq t<3 \\ \frac{322-(x+t)}{101}-\frac{\epsilon}{2}, & 220<x<320,1<t<2 \\ \frac{323-(x+t)}{101}-\frac{\epsilon}{2}, & 220<x<320,2 \leq t<3 \\ 0, & \text { otherwise }\end{cases}
$$

and

$$
\nu(x, t)= \begin{cases}\frac{222-(x+t)}{101}-\frac{\epsilon}{2}, & 120<x \leq 220,1<t<2 \\ \frac{223-(x+t)}{101}-\frac{\epsilon}{2}, & 120<x \leq 220,2 \leq t<3 \\ \frac{x+t-(221)}{101}-\frac{\epsilon}{2}, & 220<x<320,1<t<2 \\ \frac{x+t-(222)}{101}-\frac{\epsilon}{2}, & 220<x<320,2 \leq t<3 \\ 1-\epsilon, & \text { otherwise }\end{cases}
$$

Suppose 200,240 and 160 are the BGLs of the patient taken on the three consecutive days and there is no hesitancy $(\epsilon=0)$. Then, the membership and non-membership values of the BGL's in the TIFS Type I diabetic corresponding to the laboratory results of the patient are calculated as $\{\langle 200,0.8,0.2\rangle,\langle 240,0.8,0.2\rangle,\langle 160,0.4,0.6\rangle\}$ where $a=120, b=320, m=220, c=1, d=$ $3, m^{\prime}=2$ and $\epsilon=0.01$. The membership functions of the TIFSs Type I diabetic are depicted in Figure 4.1. Figure 4.2 is plotted for the continuous case of the TIFS Type I diabetic.

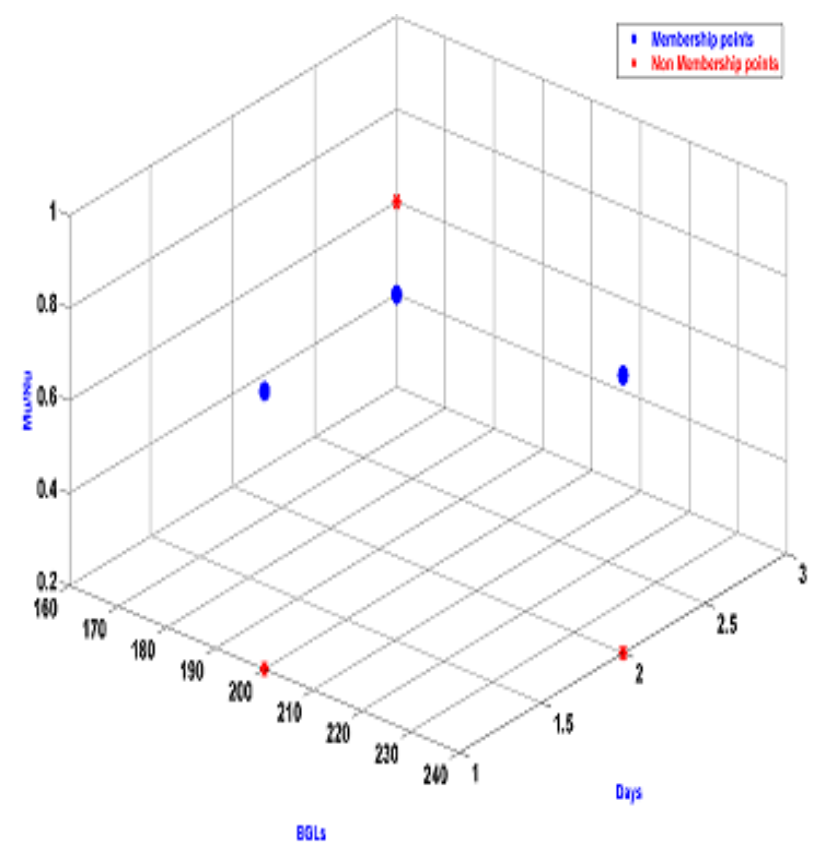

Figure 4.1. Temporal triangular fuzzification functions of the TIFS 'Type I Diabetic' for discrete data 


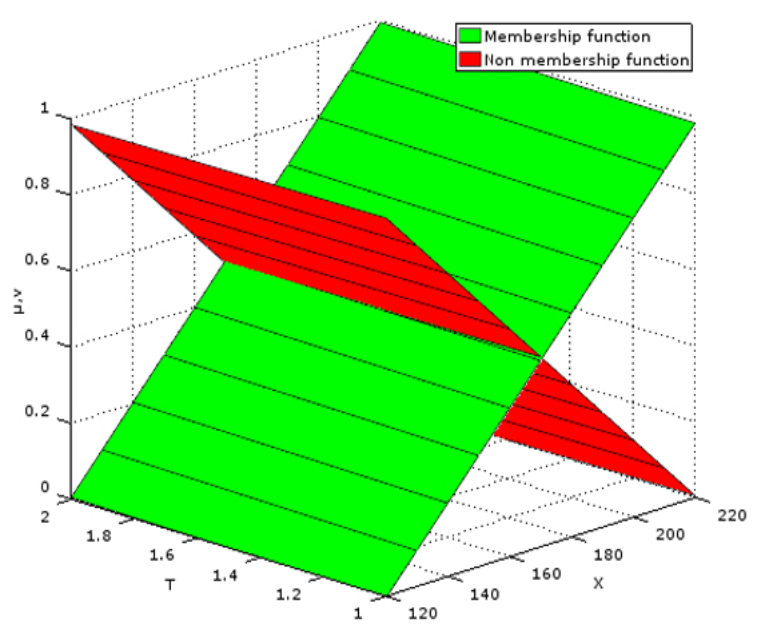

(a) $120<x \leq 220 \& 1<t<2$

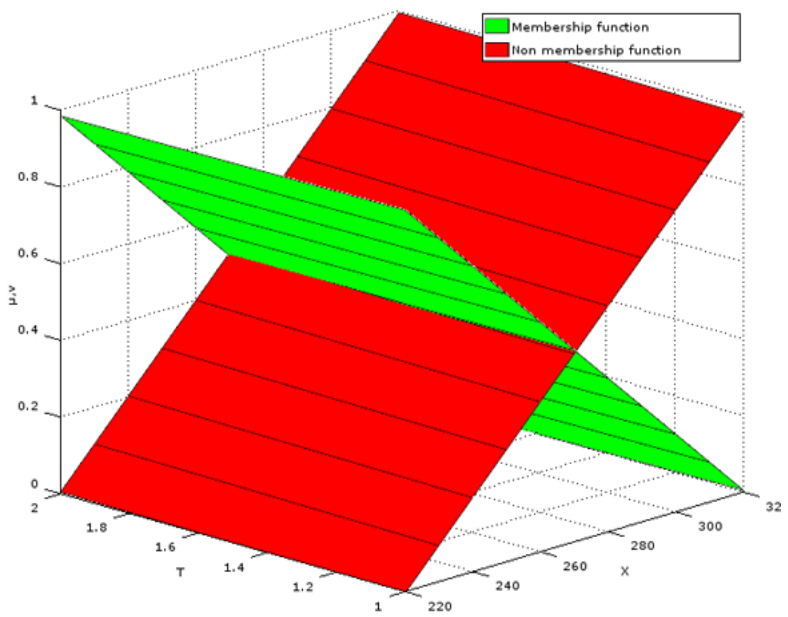

(c) $220<x<320 \& 1<t<2$

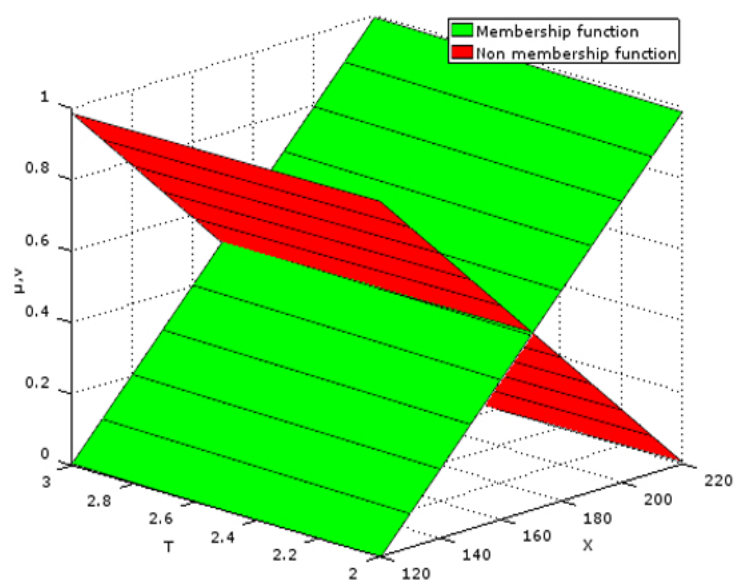

(b) $120<x \leq 220 \& 2 \leq t<3$

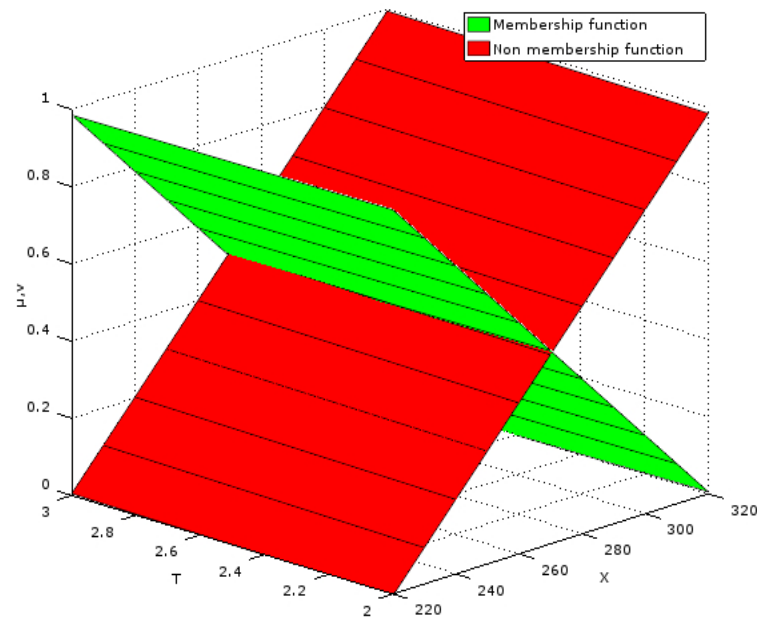

(d) $220<x<320 \& 2 \leq t<3$

Figure 4.2. Temporal triangular fuzzification functions of the TIFS 'Type I Diabetic' for continuous data

The TIFS non-diabetic can be represented by means of the temporal trapezoidal fuzzification functions defined in Section 3.2. Similarly, the TIFS low sugar (Below 50 BGL) can be defined using the temporal R-trapezoidal membership functions presented in Section 3.3, by assuming $a=50, b=80, c=1$ and $d=3$ where as the TIFS Type II diabetic is represented by using the temporal L-trapezoidal fuzzification functions defined in Section 3 where $a=280, b=400, c=1$ and $d=3$ are the suitably assumed parameters.

Example 2. Growth of bacteria in a bacterial culture is determined by the number of increase in the bacterial population which usually occurs in a geometric or exponential manner. The four characteristic phases in the growth cycle of bacteria are lag phase, exponential phase (log phase), stationary phase and death phase. In particular, the exponential phase of growth is a pattern of balanced growth in which the cells divide by binary fission in a regular manner. Consider the 
example of exponential growth of bacteria in a bacteria culture. Suppose the bacteria culture starts with 1000 bacteria and the growth increases to 2500 after two hours. The growth can be represented as a TIFS using the temporal Gaussian fuzzification functions. Let the fuzzification factor $m$ be 2 . Using the mean value $c=1750, c^{\prime}=1.5$ and standard deviation $k=1060$, $k^{\prime}=0.7$, the TIFS growth is represented by the following temporal Gaussian fuzzification functions:

$$
\mu(x, t)=\exp \left(-\frac{((x+t)-(1751.5))^{m}}{2(1060.7)^{2}}\right)-\frac{\epsilon}{2}
$$

and

$$
\nu(x, t)=1-\exp \left(-\frac{((x+t)-(1751.5))^{m}}{2(1060.7)^{2}}\right)-\frac{\epsilon}{2}
$$

The three dimensional view of the membership and non-membership functions are shown in Figure 4.3.

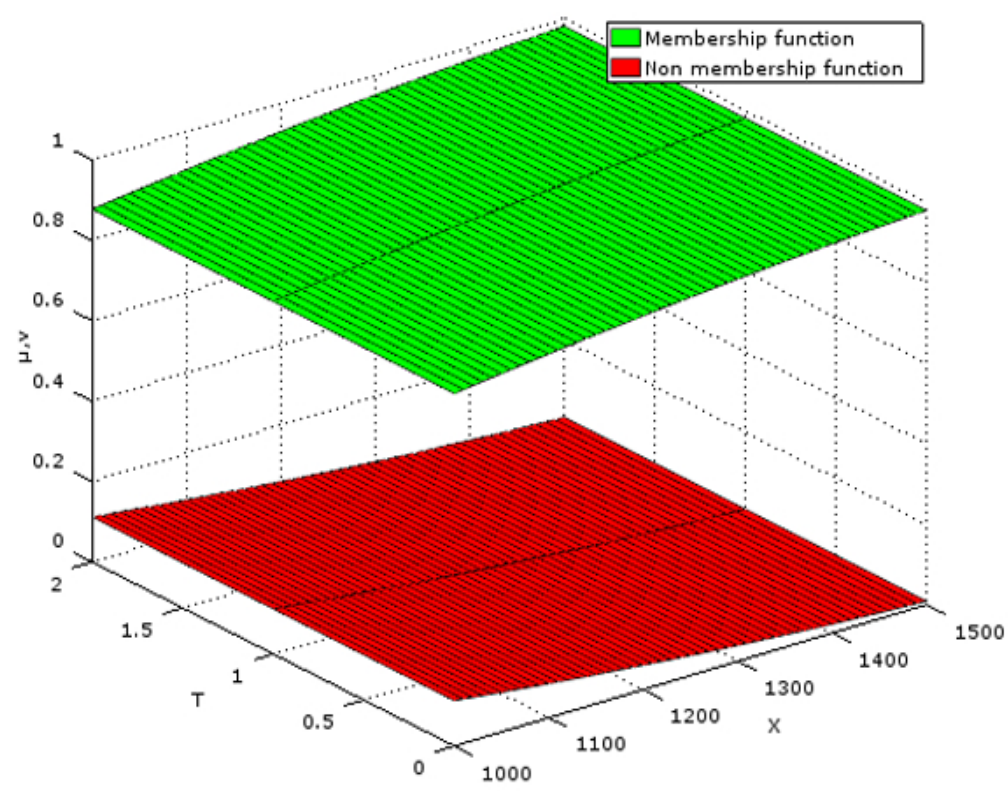

Figure 4.3. Temporal Gaussian fuzzification functions of the TIFS 'Growth'

\section{Conclusion}

In this paper, an attempt has been made to establish some standard temporal intuitionistic fuzzification functions for TIFSs which could convert an uncertain temporal data into TIFSs. Suitable examples are considered to explain the proposed functions. Further, the authors proposed to define defuzzification functions for TIFSs which will ultimately be helpful in constructing temporal intuitionistic fuzzy logic controller. 


\section{References}

[1] Atanassov, K. (1983). Intuitionistic fuzzy sets, VII ITKR's session, Sofia, June 1983 (Deposed in central Science-Technical Library of Bulg. Academy of Science, 1697/84) (in Bulgarian). Reprinted: Int. J. Bioautomation, 2016, 20(S1), S1—S6 (in English).

[2] Atanassov, K. T. (1986). Intuitionistic fuzzy sets, Fuzzy Sets and Systems, 20, 87-96.

[3] Atanassov, K. T. (1999). Intuitionistic Fuzzy Sets: Theory and Applications, Physica-Verlag, Heidelberg.

[4] Atanassov, K. T. (2012). On Intuitionistic Fuzzy Sets Theory, Springer, Berlin, Heidelberg.

[5] Atanassov, K. T., Szmidt, E. \& Kacprzyk, J. (2008). On intuitionistic fuzzy multidimensional sets, Issues in Intuitionistic Sets and Generalized Nets, 7, 1-6.

[6] Atanassov, K. T., Szmidt, E., Kacprzyk, J.,\& Rangasamy, P. (2008). On intuitionistic fuzzy multi-dimensional sets. Part 2. Advances in Fuzzy Sets, Intuitionistic Fuzzy Sets, Generalized Nets and Related Topics., Vol. I: Foundations, Academic Publishing House EXIT, Warsaw, 43-51.

[7] Atanassov, K. T., Szmidt, E.,\& Kacprzyk, J. (2010). On intuitionistic fuzzy multidimensional sets. Part 3. Developments in Fuzzy Sets, Intuitionistic Fuzzy Sets, Generalized Nets and Related Topics. Vol. I: Foundations, SRI Polish Academy of Sciences, Warsaw, 19-26.

[8] Atanassov, K. T., Szmidt, E., \& Kacprzyk, J. (2011). On intuitionistic fuzzy multidimensional sets. Part 4. Notes on Intuitionistic Fuzzy Sets, 17 (2), 1-7.

[9] Atanassova, V., \& Sotirov, S. (2012). A new formula for de-i-fuzzification of intuitionistic fuzzy sets, Notes on Intuitionistic Fuzzy Sets, 18 (3), 49-51.

[10] Ban, A., Kacprzyk, J., \& Atanassov, K. T. (2008). On de-I-fuzzification of intuitionistic fuzzy sets, Comptes Rendus de l'Academie bulgare des Sciences, 61 (12), 1535-1540.

[11] Parvathi, R., \& Radhamani, C. (2016). Multi-parameter temporal intuitionistic fuzzy sets, Notes on Intuitionistic Fuzzy Sets, 22 (1), 35-47.

[12] Radhika, C., \& Parvathi, R. (2016). Intuitionistic fuzzification functions, Global Journal of Pure and Applied Mathematics, 12 (2), 1211-1227.

[13] Ross, T. J. (2011). Fuzzy Logic with Engineering Applications, Wiley India Private Limited.

[14] Zadeh, L. A. (1965). Fuzzy sets, Information and Control, 8, 338-353. 